\title{
ON THE UNIQUENESS OF ALGEBRAIC LIMIT CYCLES FOR QUADRATIC POLYNOMIAL DIFFERENTIAL SYSTEMS WITH TWO PAIRS OF EQUILIBRIUM POINTS AT INFINITY
}

\author{
JAUME LLIBRE AND CLAUDIA VALLS
}

\begin{abstract}
Algebraic limit cycles in quadratic polynomial differential systems started to be studied in 1958, and few years later the following conjecture appeared: Quadratic polynomial differential systems have at most one algebraic limit cycle.

We prove that for a quadratic polynomial differential system having two pairs of diametrally opposite equilibrium points at infinity, has at most one algebraic limit cycle. Our result provides a partial positive answer to this conjecture.
\end{abstract}

\section{Introduction AND STATEMENT OF THE MAIN RESUlts}

Let $\mathbb{R}[x, y]$ be the ring of all real polynomials in the variables $x$ and $y$. Differential systems of the form

$$
\frac{d x}{d t}=\dot{x}=P(x, y), \quad \frac{d y}{d t}=\dot{y}=Q(x, y),
$$

where $P, Q \in \mathbb{R}[x, y]$ with $t$ real are called real polynomial differential systems. We say that system (1) has degree $m$ if the maximum degree of the polynomials $P$ and $Q$ is $m$. When $m=2$, system (1) is called a quadratic system.

System (1) has

$$
\mathcal{X}=P(x, y) \frac{\partial}{\partial x}+Q(x, y) \frac{\partial}{\partial y}
$$

as its polynomial vector field.

The algebraic curve $g(x, y)=0$ with $g=g(x, y) \in \mathbb{R}[x, y]$ is an invariant algebraic curve of the $\mathcal{X}$ if for some polynomial $K \in \mathbb{R}[x, y]$, we have

$$
\mathcal{X} g=P \frac{\partial g}{\partial x}+Q \frac{\partial g}{\partial y}=K g
$$

The polynomial $K=K(x, y)$ is called the cofactor of $g$. We recall that $g=0$ is invariant by $\mathcal{X}$.

We say that an invariant algebraic curve $g=0$ is irreducible when $g$ is irreducible in $\mathbb{R}[x, y]$. An isolated periodic orbit in the set of periodic orbits

2010 Mathematics Subject Classification. Primary: 37D99.

Key words and phrases. algebraic limit cycle, quadratic polynomial differential system, quadratic polynomial vector field. 
of $\mathcal{X}$ is called a limit cycle. An oval of an irreducible invariant algebraic curve of $\mathcal{X}$ of degree $n$ which is a limit cycle is called an algebraic limit cycle.

The following problem is a simpler version of the second part of Hilbert's 16th problem (see [17]): Let $\Sigma_{m}$ be the set of all real polynomial vector fields (2) of degree $m$ having invariant algebraic curves. Is there a uniform upper bound on the number of algebraic limit cycles of any polynomial vector field of $\Sigma_{m}$ ?

A positive answer was given in [21] where the authors consider the case in which all the invariant algebraic curves $g_{j}=0$ of $\Sigma_{m}$ are generic, i.e., they satisfy the following assumptions:

- $g_{j}=0$ has not points such that $g_{j x}=g_{j}=g_{j y}=0$,

- $g_{j}$ have no repeated factors,

- if two curves intersect at a point in the affine plane they are transversal at this point,

- there are no more than two curves $g_{j}=0$ meeting at any point in the affine plane, and

- there are no two curves having a common factor in the highest order homogeneous terms.

More precisely, they proved that for these generic curves, the number of limit cycles is $1+(m-1)(m-2) / 2$ if $m$ is even and $(m-1)(m-2) / 2$ if $m$ is odd, and these bounds are achieved. For other related papers treating this problem, see [22] and [29]. However there are plenty of invariant algebraic curves that do not satisfy these generic conditions, so for $\Sigma_{2}$ it remains to solve the following open problem: to know if when the invariant algebraic curves of a quadratic polynomial differential system do not satisfy these generic conditions, this system also has at most one algebraic limit cycle.

In [27] it is proved that quadratic polynomial vector fields can have algebraic limit cycles of degree 2 , and that they are unique whenever they exist. In $[13,14,15]$ the author proved that quadratic vector fields do not have algebraic limit cycles of degree 3 (see also [6, 18, 25, 28] for different and shorter proofs). In [26] it is found the first class of algebraic limit cycles of degree 4 inside the quadratic vector fields. The second class was found in [16]. More recently, two new classes have been found and in [9] the authors proved that there are no other algebraic limit cycles of degree 4 for quadratic vector fields. The uniqueness of these limit cycles was proved in [11]. It is known that there are quadratic polynomial differential systems having algebraic limit cycles of degree 5 and 6 , see [9], and that this limit cycle is the unique one for these differential systems. Other results on algebraic limit cycles can be found in [20].

It turns out that the open problem mentioned above is too hard to deal with and that is why a simpler version of this problem has kept the attention of the researchers for many years. Thus the following conjecture appeared explicitly in $[19,22]$, but was known many years before among the mathematicians working in this subject. 
In [24] the authors proved the conjecture in the case in which the quadratic polynomial differential systems have only one pair of equilibrium points at infinity. So it is still open the conjecture in the cases in which the system has two or three pairs of diametrally opposite equilibrium points at infinity. Here we solve the conjecture when the quadratic differential systems have two pairs of diametrally opposite equilibrium points at infinity.

Theorem 1. Quadratic polynomial differential systems with at most two pairs of equilibrium points at infinity have at most one algebraic limit cycle.

The proof of Theorem 1 is divided in two sections 3 and 4 . In section 2 we state some known facts about quadratic polynomial differential systems that we shall need and that are well known. We have also added an appendix where we state an auxiliary result that we shall need but that is not known.

\section{QUADRATIC SYSTEMS: KNOWN RESULTS}

The following theorems are well known.

The next result is proved in $[5,12]$.

Theorem 2. Quadratic polynomial differential systems having an invariant algebraic straight line have at most one limit cycle.

For a proof of the next result see $[11,6]$.

Theorem 3. Quadratic polynomial differential systems having an algebraic limit cycle of degree two or four have at most one limit cycle.

In the papers $[6,18,25,28]$ there are different proofs of the next result.

Theorem 4. Quadratic polynomial differential systems have no algebraic limit cycles of degree 3 .

In view of Theorems 3 and 4 from now on we will consider algebraic limit cycles of degree $n \geq 5$.

The next result is Proposition 8 of [23].

Proposition 5. All finite equilibrium points of a quadratic polynomial differential system (3) and all points satisfying $\partial g / \partial x=\partial g / \partial y=0$ of an invariant algebraic curve $g=0$ are contained in the union of $\{K=0\}$ and $\{g=0\}$, where $K$ is the cofactor of $g=0$.

From Theorem 4 of [23] and its proof and Theorem 2 of [24] we have:

Theorem 6. If a quadratic polynomial differential system has an algebraic limit cycle of degree $n$, then it can be transformed, through an affine change of variables and a scaling of the time, into one of the following two systems. First

$$
\begin{aligned}
& \dot{x}=\xi x-y+a x^{2}+b x y, \\
& \dot{y}=x-\xi y+d x^{2}+e x y+f y^{2},
\end{aligned}
$$


with $d \neq 0$ and $\xi \in(-1,1)$, and second

$$
\begin{aligned}
& \dot{x}=-y+a x^{2}+b x y+c y^{2}, \\
& \dot{y}=x+e x y+f y^{2} .
\end{aligned}
$$

The cofactor of $g=0$ in both cases is ny.

It is pointed out in [23] that if systems (3) and (4) have a limit cycle, it must surround the origin. The next result is Proposition 13 of [23].

Proposition 7. Let $P_{2}$ and $Q_{2}$ be the homogeneous components of $P$ and $Q$, respectively. If $y P_{2}-x Q_{2} \equiv 0$, then the quadratic system has no limit cycles.

Sometimes we shall use the extension of the real polynomial differential system to the complex projective space for obtaining additional information on the real system. For more details on this extension see for instance $[5,6]$. The following result is Lemma 11 of [23].

Proposition 8. The complex invariant algebraic curve $g=0$ must intersect the infinity at least in one point, eventually complex. All the intersection points must be equilibrium points of the extended vector field in the complex projective space.

The following lemma due to Christopher [8] says how must be the higher degree terms of an invariant algebraic curve $g=0$ of a polynomial differential system (1).

Proposition 9. Suppose that a polynomial differential system (1) of degree 2 has the invariant algebraic curve $g=0$ of degree $n$. Let $P_{2}, Q_{2}$ and $g_{n}$ be the homogeneous components of $P, Q$ and $g$ of degree 2 and $n$ respectively. Then the irreducible factors of $g_{n}$ must be factors of $y P_{2}-x Q_{2}$.

The proof of the next theorem can be found in [7] (see also Theorem A2 in [10]).

Theorem 10. System

$$
\dot{x}=-y+a_{1} x+a_{2} x^{2}, \quad \dot{y}=x\left(1+a_{3} x+a_{4} y\right),
$$

has at most one limit cycle surrounding the origin.

The proof of the next theorem can be found in [24] (see Theorem 2).

Theorem 11. A quadratic polynomial differential system with at most one pair of equilibrium points at infinity, has at most one limit cycle.

In view of Theorem 11 in order to prove Theorem 1 we can restrict ourselves to study the quadratic polynomial differential systems having two pairs of equilibrium points at infinity.

We now give necessary conditions in order that a polynomial be a cofactor of an invariant algebraic curve.

Let $g(x, y)=0$ be an invariant algebraic curve of a planar polynomial differential equation of the form $\dot{x}=P(x, y), \dot{y}=Q(x, y)$ where $P, Q$ are 
polynomials of degree $m$, and $\left(x_{0}, y_{0}\right)$ a point such that $g\left(x_{0}, y_{0}\right)=0$. We may expand $g(x, y)$ in powers of $x-x_{0}$ and $y-y_{0}: g(x, y)=g_{s}(x, y)+$ $g_{s+1}(x, y)+\cdots+g_{n}(x, y)$ where $n$ is the degree of $g$ and $g_{j}(x, y)$ are homogeneous polynomials of degree $j$ in powers of $x-x_{0}$ and $y-y_{0}$. Let $s$ be the lowest degree in this expansion with $g_{s}(x, y) \not \equiv 0$. Since $g\left(x_{0}, y_{0}\right)=0$ we have $s \geq 1$. As $g_{s}(x, y)$ is a homogeneous polynomial of degree $s$ in powers of $x-x_{0}$ and $y-y_{0}$ it factorizes in $s$ linear homogeneous polynomials, that is,

$g_{s}(x, y)=\ell_{1} \ell_{2} \cdots \ell_{s} \quad \ell_{i}=a_{i}\left(x-x_{0}\right)+b_{i}\left(y-y_{0}\right), \quad a_{i}, b_{i} \in \mathbb{C}, i=1,2 \ldots, s$.

We say that $g_{s}(x, y)=0$ is the equation of the tangents of the curve $g(x, y)=0$ in $\left(x_{0}, y_{0}\right)$. We will denote by $\lambda, \mu$ the eigenvalues of the linear approximation at this point $\left(x_{0}, y_{0}\right)$. The following result is proved in [3] (see Theorems 13 and 14). As usual $\mathbb{N}$ denotes the set of positive integers.

Theorem 12. We consider a system $x^{\prime}=P(x, y), y^{\prime}=Q(x, y)$ where $P, Q$ are polynomials of degree $m$ and $\left(x_{0}, y_{0}\right)$ be one of its equilibrium points and $g(x, y)=0$ be an irreducible invariant algebraic curve with cofactor $k(x, y)$. If $g\left(x_{0}, y_{0}\right) \neq 0$ then $k\left(x_{0}, y_{0}\right)=0$. Moreover, if $g\left(x_{0}, y_{0}\right)=0$, with the described notation, we have that $g_{s}(x, y)=\left(\ell_{\lambda}\right)^{r}\left(\ell_{\mu}\right)^{s-r}$ with $r, s$ non-negative integers and $r \leq s$ and

$$
k\left(x_{0}, y_{0}\right)=r \mu+(s-r) \lambda .
$$

Furthermore:

(a) if either $\mu \neq 0$ and $\lambda$ and $\mu$ are rationally independent, or $\lambda / \mu<0$, or $\mu=0$, then

- either $s=2, r=1$ and $g_{2}=\ell_{\lambda} \ell_{\mu}$;

- or $s=1$ and $g_{1}=\ell_{\lambda}$;

- or $s=1$ and $g_{1}=\ell_{\mu}$.

(b) if $\mu \neq 0$ and $\lambda$ and $\mu$ are rationally dependent and $\lambda / \mu>0$ we assume that $\lambda / \mu>1$.

- if $\lambda / \mu=d$ with $d \in \mathbb{N}$ and $d>1$, and the differential system is linearizable in $\left(x_{0}, y_{0}\right)$ then there exists a non-negative integer $r$ and $\varepsilon \in\{0,1\}$ such that $s=r+\varepsilon$ and $g_{s}=\left(\ell_{\lambda}\right)^{\varepsilon}\left(\ell_{\mu}\right)^{r}$.

- if $\lambda / \mu=d$ with $d \in \mathbb{N}, d>1$ and the differential system is non-linearizable in $\left(x_{0}, y_{0}\right)$, then $s=1$ and $g_{1}=\ell_{\lambda}$.

- if $\lambda / \mu=p / q$ with $p, q \in \mathbb{N}$ and $1<q<p$, then

* either $s=1$ and $g_{1}=\ell_{\lambda}$;

* or there exists a non-negative integer $r$ and $\varepsilon \in\{0,1\}$ such that $s=r q+\varepsilon$ and $g_{s}=\left(\ell_{\lambda}\right)^{\varepsilon}\left(\ell_{\mu}\right)^{r q}$;

* or there exists a non-negative integer $r$ and $\varepsilon \in\{0,1\}$ such that $s=r q+\varepsilon+1$ and $g_{s}=\left(\ell_{\lambda}\right)^{\varepsilon}\left(\ell_{\mu}\right)^{r q+1}$.

It is stated in [3] that Theorem 12 is also valid in $\mathbb{C P}(2)$. We recall that $\mathbb{C P}(2)=\left\{\mathbb{C}^{3} \backslash\{(0,0,0)\}\right\} / \sim$ with the equivalence relation $[X, Y, Z] \sim$ $\left[X^{*}, Y^{*}, Z^{*}\right]$ if and only if there exists $\nu \in \mathbb{C} \backslash\{0\}$ such that $\left[X^{*}, Y^{*}, Z^{*}\right]=$ $\nu[X, Y, Z]$. Consider a point $p:=\left[X_{0}, Y_{0}, Z_{0}\right] \in \mathbb{C P}(2)$, and without loss of generality we assume that $Z_{0} \neq 0$. We define the local coordinates in $p$ by $x=X / Z$, and $y=Y / Z$. So, in local coordinates we have $p=\left(x_{0}, y_{0}\right)$ 
with $x_{0}=X_{0} / Z_{0}$ and $y_{0}=Y_{0} / Z_{0}$. We consider $\tilde{P}(x, y)=Z^{m} P\left(\frac{X}{Z}, \frac{Y}{Z}\right)$ and $\tilde{Q}(x, y)=Z^{m} Q\left(\frac{X}{Z}, \frac{Y}{Z}\right)$. The coordinates $(x, y)$ are usually called finite coordinates and the set of points $[X, Y, Z] \in \mathbb{C P}(2)$ with $Z=0$ is called the line at infinity. An invariant algebraic curve $f(x, y)=0$ with cofactor $k(x, y)$ defines an invariant algebraic curve in $\mathbb{C P}(2)$ by $F(X, Y, Z)=$ $Z^{n} f(X / Z, Y / Z)$ where $n$ is the degree of $f$, and the associated cofactor is $k(X, Y, Z)=Z^{m-1} k(X / Z, Y / Z)$.

\section{Proof of Theorem 1 for system (3)}

Theorem 1 will be an immediate consequence of the proof of the following theorem.

Theorem 13. Let $g=0$ be an invariant algebraic curve of degree $n \geq 5$ of a quadratic polynomial differential system (3). Assume that $g=0$ has two pairs of equilibrium points at infinity. Then there is at most one limit cycle on $g=0$.

Proof. Let $g=\left(\sum_{i=0}^{m} g_{n-i, i} x^{n-i} y^{i}\right)+\cdots$, with $0 \leq m \leq n$ and $g_{n-m, m} \neq 0$, where the dot denotes the terms of order $n-1$ and lower. The coefficient of the term $x^{n-m} y^{m+1}$ in the expression of $\dot{g}-n y g=0$ is equal to

$$
g_{n-m, m}((n-m) b+m f-n)=0 .
$$

Therefore

$$
b=(n-m f) /(n-m) \text { if } m<n \text { and } \quad f=1 \text { if } m=n .
$$

From Proposition 9 we have

$$
g_{n}=\sum_{i=0}^{m} g_{n-i, i} x^{n-i} y^{i}=x^{n-m}\left(x-x_{1} y\right)^{k}\left(x-x_{2} y\right)^{m-k},
$$

where

$$
x_{1,2}=\frac{a-e \pm \sqrt{\Delta}}{2 d} \quad \text { with } \quad \Delta=(a-e)^{2}+4 d(b-f)
$$

are the roots of the polynomial $d x^{2}-(a-e) x-(b-f)=0$. The case in which $\Delta<0$ was proved in [24] because in that case at infinity there is either a unique pair of singular points which are the endpoints of $x=0$. Here, either $x_{2}=0$ or $x_{1}=x_{2} \neq 0$ (otherwise at infinity there is a unique pair of singular points or three pairs of singular points). In the first case $f=b$ (and $\left.\Delta=(a-e)^{2}\right)$, and in the second case $f=b-(a-e)^{2} /(4 d)$ with $a \neq e($ and $\Delta=0)$.

From the proof of Theorem 5 of [23] when $m=n$ we can always assume that $g(x)=\left(x-x_{1} y\right)^{n / 2}\left(x-x_{2} y\right)^{n / 2}$ with $x_{1}=\bar{x}_{2} \notin \mathbb{R}$ being $\bar{x}_{2}$ the complex conjugate of $x_{1}$. But since from (5) $x_{1}$ and $x_{2}$ are real, we can assume $m<n$. We consider two cases: $b=f$ and $f=b-(a-e)^{2} /(4 d)$.

Case 1: $f=b$. In this case $f=b=1$. We consider two different subcases: $m=0$ and $m \geq 1$. 
Subcase 1.1: $m=0$. The terms of degree $n$ of $g$ are $g_{n}=x^{n}$. Imposing that

$$
\left(a x^{2}+x y\right) \frac{\partial g_{n}}{\partial x}+\left(d x^{2}+e x y+y^{2}\right) \frac{\partial g_{n}}{\partial y}-n y g_{n}=0
$$

we get that $n a x^{n+1}=0$ which implies $a=0$. Then system (3) becomes

$$
\dot{x}=\xi x-y+x y, \quad \dot{y}=x-\xi y+d x^{2}+e x y+f y^{2}
$$

with $f=1$. If $\xi=0$ then (7) has the invariant straight line $x=1$. In view of Theorem 2 it has at most one limit cycle. So, from now on we assume $\xi \neq 0$.

Now we compute the terms of degree $n-1$. In view of Lemma 15 we must solve the equation

$$
\left(d x^{2}+e x y+(f-1) y^{2}\right) \frac{\partial g_{n-1}}{\partial y}+n(\xi x-y) x^{n-1}-y g_{n-1}=0 .
$$

Moreover setting $g_{n-1}=-n x^{n-1}+h_{n-1}$ we rewrite (15) for $h_{n-1}$ in the form

$$
\left(d x^{2}+e x y+(f-1) y^{2}\right) \frac{\partial h_{n-1}}{\partial y}+n \xi x^{n}-y h_{n-1}=0 .
$$

We recall that $f=1$ and we consider different cases.

Subcase 1.1.1: $e=0$. In this case

$$
h_{n-1}=-e^{y^{2} /\left(2 d x^{2}\right)}\left(\frac{\xi n \sqrt{\pi}}{\sqrt{2 d}} x^{n-1} \operatorname{Erf}\left(\frac{y}{\sqrt{2 d} x}\right)+K(x)\right),
$$

where $K(x)$ is any function in the variable $x$ and Erf is the error function (see [1] for more details). Taking into account that $h_{n-1}$ must be a homogeneous polynomial of degree $n-1$ in the variables $x$ and $y$ and that $\xi \neq 0$ we get a contradiction.

Subcase 1.1.2: $e \neq 0$. In this case we have

$$
h_{n-1}=e^{y /(e x)}\left(e^{d / e^{2}} \xi n x^{n-1} E_{1-\frac{d}{e^{2}}}\left(\frac{d x+e y}{e^{2} x}\right)+(d x+e y)^{-d / e^{2}} K(x)\right),
$$

where $K(x)$ is any function in the variable $x$ and $E_{n}$ is the exponential integral function (see [1] for details, which can be written as a series). Since $\xi n \neq 0$, the function $h_{n-1}$ is never a homogeneous polynomial of degree $n-1$ in the variables $x$ and $y$. Hence, this case is not possible.

Subcase 1.2: $m \geq 1$. Then $g_{n}=x^{n-m}(x-(a-e) y / d)^{m}$. In this case imposing (6) we get that $e=a(m-n) / m$. Note that $a \neq 0$ otherwise $e=0$ which is not possible because system (3) would have a unique pair of diametrally opposite equilibrium points at infinity. The terms of degree $n$ of $g$ are $g_{n}=x^{n-m}(x-a n y /(d m))^{m}$.

If we pass to the coordinates $[X, Y, Z]$, the equilibrium points at infinity have all of them coordinate $Y_{0} \neq 0$, so the local coordinates $(u, v)$ with $u=X / Y$ and $v=Z / Y$ are taken at these points in order to apply Theorem 
12. Then system (3) with $f=b=1$ and $e=a(m-n) / m$ in coordinates $(u, v)$ becomes

$$
\begin{aligned}
& \dot{u}=\frac{1}{m}\left(a n u^{2}-d m u^{3}-m v+2 \xi m u v-m u^{2} v\right), \\
& \dot{v}=-\frac{v}{m}\left(m+a m u-a n u+d m u^{2}-\xi m v+m u v\right),
\end{aligned}
$$

and

$$
g(u, v)=u^{n-m}(u-a n /(d m))^{m}+v g_{n-1}(u)+v^{2} g_{n-2}(u)+\cdots+v^{n} g_{0}(u),
$$

where $g_{j}(u)$ are polynomials in the variable $u$ of degree $j$, and the cofactor becomes

$$
k(u, v)=n .
$$

The infinite equilibrium points for system (3) are on $v=0$ and $u^{2}(d m u-$ $a n)=0$ that is

$$
v=0 \text { with } u=0 \text { (double point) or } u=\frac{a n}{d m} \text { (simple point). }
$$

We take the double point $u=v=0$. Since $m<n$ we have that $g(0,0)=0$. Moreover the eigenvalues of the Jacobian matrix at that point are

$$
\mu=0, \quad \lambda=-1 .
$$

Since $\mu=0$, it follows from Theorem 12 (a) that either $s=2$ and $r=1$ in which case $k(0,0)=\lambda$, or $s=1$ and $r=0$ in which case $k(0,0)=\lambda$, or $s=1, r=1$ in which case $k(0,0)=0$. Since $k(0,0)=n$ we must have either $n=0$ or $n=-1$ which are both not possible. So, this case is not possible.

Case 2: $f=b-(a-e)^{2} /(4 d)$. In this case

$$
f=1+\frac{e^{2} n(n-m)}{d(m-2 n)^{2}} \quad \text { and } \quad b=1-\frac{e^{2} m n}{d(m-2 n)^{2}} .
$$

We consider two different subcases.

Subcase 2.1: $m=0$. In this case $f=1+e^{2} /(4 d)$ and $b=1$. Imposing that

$$
\left(a x^{2}+x y\right) \frac{\partial g_{n}}{\partial x}+\left(d x^{2}+e x y+\left(1+\frac{e^{2}}{4 d}\right) y^{2}\right) \frac{\partial g_{n}}{\partial y}-n y g_{n}=0,
$$

we get that $a=0$.

We recall that system (3) in this case becomes (7) with $f=1+e^{2} /(4 d)$. Again, if $\xi=0$ then (7) has the invariant straight line $x=1$ and so it has at most one limit cycle. So, from now on we assume $\xi \neq 0$.

The terms of degree $n$ of $g$ are $g_{n}=x^{n}$. Now computing the terms of degree $n-1$ and proceeding as in case 1.1 we can write $g_{n-1}=-n x^{n-1}+h_{n-1}$ where $h_{n-1}$ must satisfy (9) with $f=1+e^{2} /(4 d)$. Note that since $f \neq 1$ we must have $e \neq 0$. In this case, setting $\mathcal{E}_{e, f}(x, y)=e x+2(f-1) y$ we have

$$
\begin{aligned}
h_{n-1} & =\exp \left(\frac{e x}{(f-1) \mathcal{E}_{e, f}(x, y)}\right)\left(-\frac{2 \xi n x^{n}}{\mathcal{E}_{e, f}(x, y)} E_{1 /(1-f)}\left(\frac{e x}{(f-1)\left(\mathcal{E}_{e, f}(x, y)\right.}\right)\right. \\
& \left.+K(x)\left(\mathcal{E}_{e, f}(x, y)\right)^{1 /(f-1)}\right),
\end{aligned}
$$


where $E_{n}$ is the exponential integral function and $K(x)$ is any function in the variable $x$. Since $\xi e \neq 0$, the function $h_{n-1}$ is never a homogeneous polynomial of degree $n-1$ in the variables $x$ and $y$ and so this case is not possible.

Case 2.2: $m \geq 1$ In this case imposing that

$$
\left(a x^{2}+b x y\right) \frac{\partial g_{n}}{\partial x}+\left(d x^{2}+e x y+f y^{2}\right) \frac{\partial g_{n}}{\partial y}-n y g_{n}=0,
$$

with $f$ and $b$ as in (11), we get that $e=a(m-2 n) / m$. Note that $a \neq 0$ otherwise we would be in the case $m=0$.

The terms of degree $n$ of $g$ are $g_{n}=x^{n-m}(x-a n y /(d m))^{m}$. Note that now

$$
b=1-\frac{a^{2} n}{d m}, \quad e=\frac{a(m-2 n)}{m} \text { and } \quad f=1+\frac{a^{2} n(n-m)}{d m^{2}} .
$$

If we pass to the coordinates $[X, Y, Z]$, the equilibrium points at infinity have all of them coordinate $Y_{0} \neq 0$ so local coordinates $(u, v)$ with $u=X / Y$ and $v=Z / Y$ are taken at these points in order to apply Theorem 12 . System (3) with $f, b, e$ as in (12) becomes

$$
\begin{aligned}
\dot{u}= & -\frac{1}{d m^{2}}\left(a^{2} n^{2} u-2 a d m n u^{2}+d^{2} m^{2} u^{3}+d m^{2} v-2 d \xi m^{2} u v+d m^{2} u^{2} v\right), \\
\dot{v}= & -\frac{v}{d m^{2}}\left(d m^{2}-a^{2} m n+a^{2} n^{2}+a d m^{2} u-2 a d m n u+d^{2} m^{2} u^{2}\right. \\
& \left.-d \xi m^{2} v+d m^{2} u v\right),
\end{aligned}
$$

and

$$
g(u, v)=u^{n-m}(u-a n /(d m))^{m}+v g_{n-1}(u)+v^{2} g_{n-2}(u)+\cdots+v^{n} g_{0}(u),
$$

where $g_{j}(u)$ are polynomials in the variable $u$ of degree $j$, and the cofactor becomes

$$
k(u, v)=n .
$$

The equilibrium points for system (3) are on $v=0$ and

$$
u=0 \text { (single point) or } u=\frac{a n}{d m} \text { (double point). }
$$

We take the double point $(u, 0)$ with $u=a n /(d m)$. Since $m \geq 1$ we have that $g\left(\frac{a n}{d m}, 0\right)=0$. Moreover the eigenvalues of the Jacobian matrix at that point are

$$
\mu=0, \quad \lambda=-1 .
$$

Since $\mu=0$, it follows from Theorem 12 (a) that either $s=2$ and $r=$ 1 in which case $k(a n /(d m), 0)=\lambda$, or $s=1$ and $r=0$ in which case $k(a n /(d m), 0)=\lambda$, or $s=1, r=1$ in which case $k(a n /(d m), 0)=0$. Since $k(a n /(d m), 0)=n$ we must have either $n=0$ or $n=-1$ which are both not possible. So, this case is not possible.

Proof of Theorem 1 for system (3). In view of the proof of Theorem 13, the unique possible case to have an algebraic limit cycle leads to a system (3) having at most one limit cycle. This concludes the proof in this case. 


\section{Proof of Theorem 1 for System (4)}

The proof of Theorem 1 for system (4) will be an immediate consequence of the proof of the following theorem.

Theorem 14. Let $g=0$ be an invariant algebraic curve of degree $n \geq 5$ of a quadratic polynomial differential system (4). Assume that $g=0$ has two pairs of equilibrium points at infinity. Then there is at most one limit cycle on $g=0$.

Proof. Let $g=\left(\sum_{i=0}^{m} g_{n-i, i} y^{n-i} x^{i}\right)+\cdots$ with $g_{n-m, m} \neq 0$, where the dots indicate terms of degree $n-1$ and lower. The coefficient of the term $y^{n-m} x^{m+1}$ in the expression $\dot{g}-n y g$ is equal to $a m+e(n-m)=0$. Therefore

$$
e=\frac{a m}{m-n} \text { if } m \neq n, \quad \text { and } \quad a=0 \text { if } m=n .
$$

By Propositions 7 and 9 (we are assuming that the line at infinity is not formed by equilibrium points, otherwise the system cannot have a limit cycle), we have that $\sum_{i=0}^{m} g_{n-i, i} y^{n-i} x^{i}=y^{n-m}\left(y-y_{1} x\right)^{k}\left(y-y_{2} x\right)^{m-k}$ with $0 \leq k \leq m$, where $y_{1}, y_{2}$ are the roots of $c y^{2}+(b-f) y+(a-e)=0$.

We now consider different cases: $m=n$ and $m<n$.

Case 1: $m=n$. In this case $a=0$. Proceeding as in [23], there are three reasons for having the condition $m=n$. First we simply have chosen the wrong system of coordinates, and there is some other real singular point of the system at infinity through which $g=0$ passes. In that case the system can be transformed into system (3) with $m<n$.

The second reason for having $m=n$ is that all the branches of $g=0$ go through non-real equilibrium points of the system at infinity. This means that $y_{1}=\overline{y_{2}} \notin \mathbb{R}$. In that case system (4) would have only a pair of equilibrium points at infinity and in view of Theorem 11 it is proved that in this case the system has at most one limit cycle.

The third reason for having $m=n$ is that $c=0$. Then system (4) becomes

$$
\dot{x}=-y+b x y, \quad \dot{y}=x+e x y+f y^{2} .
$$

If $b \neq 0$ system (4) has the invariant straight line $x=1 / b$ and in view of Theorem 2 system (4) has at most one limit cycle. So, $b=0$. Moreover, if $f=0$, then either the system has no equilibrium points at infinity (if $e \neq 0$ ) or the line at infinity is formed by equilibrium points (if $e=0$ ). In both cases it follows, respectively, from Propositions 8 and 7 that system (4) has no limit cycles.

In short, $b=0$ and $f \neq 0$. In this case $g_{n}=(y-e x / f)^{n}$. Imposing that

$$
\left(e x y+f y^{2}\right) \frac{\partial g_{n}}{\partial y}-n y g_{n}=0
$$


we get

$$
y\left(y-\frac{e}{f} x\right)^{n-1}\left(\frac{e(f+1)}{f} x+(f-1) y\right)=0,
$$

and so

$$
f=1 \text { and } e=0 .
$$

It follows from Theorem 10 (making the change $x \rightarrow y, y \rightarrow x, t \rightarrow-t$ ) that system (4) has at most one limit cycle.

Case 2: $m<n$. If $a=0$ then $e=0$ and it follows from Theorem 10 (making the change $x \rightarrow y$ and $y \rightarrow x$ ) that system (4) has at most one limit cycle.

We can thus assume that $a \neq 0$ and so $e=a m /(m-n)$. We consider two cases.

Subcase 2.1: $m=0$. In this case $e=0$ and $g_{n}=y^{n}$. Imposing that

$$
f y^{2} \frac{\partial g_{n}}{\partial y}-n y g_{n}=0
$$

we get $f=1$. Since $e=0$ and $f=1$, system (4) becomes

$$
\dot{x}=-y+a x^{2}+b x y+c y^{2}, \quad \dot{y}=x+y^{2} .
$$

The terms of degree $n$ of $g$ are $g_{n}=y^{n}$. Now we compute the terms of degree $n-1$. In view of Lemma 15 we must solve the equation

$$
\left(a x^{2}+(b-1) x y+c y^{2}\right) \frac{\partial g_{n-1}}{\partial x}+n x y^{n-1}=y g_{n-1} .
$$

We show that $g_{n-1}=y^{n-2} R_{1}$ with $R_{1}=a_{1} x+b_{1} y$. Indeed, if we set $y=0$ in (14) and denote by $\bar{g}_{n-1}=g_{n-1}(x, 0)$ we have that

$$
a x^{2} \frac{d \bar{g}_{n-1}}{d x}=0,
$$

which means that $g_{n-1}=y R_{n-2}$ where $R_{n-2}$ is a homogeneous polynomial of degree $n-2$ which satisfies, after simplifying by $y$,

$$
\left(a x^{2}+(b-1) x y+c y^{2}\right) \frac{\partial R_{n-2}}{\partial x}+n x y^{n-2}=y R_{n-2} .
$$

Proceeding inductively we get that $g_{n-1}=y^{n-2} R_{1}$ with $R_{1}=a_{1} x+b_{1} y$ and $R_{1}$ satisfies

$$
\left(a x^{2}+(b-1) x y+c y^{2}\right) a_{1}+n x y=y\left(a_{1} x+b_{1} y\right) .
$$

So equating the terms with $x^{2}$ we get $a a_{1}=0$. Since $a \neq 0$ we must have $a_{1}=0$. Then $n x y=b_{1} y^{2}$ which is not possible. Hence, this case is not possible.

Subcase 2.2: $m \geq 1$ We consider two different subcases $c=0$ and $c \neq 0$.

Subcase 2.2.1: $c=0$. Since there is at most two pairs of singular points at infinity we must have that either $a=e$ or $b=f$ because the pairs of singular points at infinity are the endpoints of the straight lines defined by the real 
linear factors of $x y((a-e) x+(b-f) y)$. Since $e=a m /(m-n)$ and $a \neq 0$ we must have $b=f$. Then $g_{n}=y^{n-m} x^{m}$. Imposing that $g_{n}$ satisfies

$$
\left(a x^{2}+b x y\right) \frac{\partial g_{n}}{\partial x}+\left(\frac{a m}{m-n} x y+b y^{2}\right) \frac{\partial g_{n}}{\partial y}=n y g_{n}
$$

we get

$$
y^{n-m} x^{m} n(b-1)=0 .
$$

Therefore $b=1$. Then $b=f=1$ and system (4) becomes

$$
\dot{x}=-y+a x^{2}+x y, \quad \dot{y}=x+\frac{a m}{m-n} x y+y^{2} .
$$

Now we compute the terms of degree $n-1$. In view of Lemma 15 we must solve the equation

$$
\begin{aligned}
& a x^{2} \frac{\partial g_{n-1}}{\partial x}+\frac{a m}{m-n} x y \frac{\partial g_{n-1}}{\partial y}-m x^{m-1} y^{n-m+1} \\
& \quad+(n-m) x^{m+1} y^{n-m-1}=y g_{n-1} .
\end{aligned}
$$

Assume $g_{n-1}$ is not divisible by $y$. Then, evaluating (15) on $y=0$ and setting $\bar{g}_{n-1}=\bar{g}_{n-1}(x)=g_{n-1}(x, 0)$ (must be of the form $\alpha x^{n-1}$ with $\alpha \neq 0$ ) we get

$$
a x^{2} \frac{d \bar{g}_{n-1}}{d x}=a \alpha x^{n}=0 \quad \text { that is } \quad \alpha=0,
$$

which is not possible. Thus $g_{n-k}$ must be divisible by $y$. We write it as $g_{n-1}=y^{\ell} h_{n-1-\ell}$ for some $\ell>0$, being $h_{n-1-\ell}$ a homogeneous polynomial of degree $n-1-\ell$. We consider that $\ell<n-m-1$. Then $h_{n-1-\ell}$ satisfies, after simplifying by $y^{\ell}$

$$
\begin{aligned}
& a x^{2} \frac{\partial h_{n-1-\ell}}{\partial x}+\frac{a m}{m-n} x y \frac{\partial h_{n-1-\ell}}{\partial y}-m x^{m-1} y^{n-m+1-\ell} \\
& \quad+(n-m) x^{m+1} y^{n-m-1-\ell}=\left(y+a \frac{\ell m}{n-m} x\right) h_{n-1-\ell} .
\end{aligned}
$$

Evaluating it on $y=0$ and setting $\bar{h}_{n-1-\ell}=h_{n-1-\ell}(x, 0)=\beta_{0} x^{n-1-\ell}$ (we recall that $h_{n-1-\ell}$ is a homogeneous polynomial of degree $n-1-\ell$ ) we must have

that is

$$
a \beta_{0}(n-1-\ell) x^{n-1-\ell}=a \beta_{0} \frac{\ell m}{n-m} x^{n-1-\ell}
$$

$$
a \beta_{0}\left(n-1-\ell-\frac{\ell m}{n-m}\right) x^{n-1-\ell}=0 .
$$

Since $a \neq 0$ then either $\beta_{0}=0$ or $n-1-\ell-\ell m /(n-m)=0$. That is $n(n-m)-(n-m)-\ell n=0$. Note that since $\ell<n-1-m$ we have

$$
n(n-m)-(n-m)-\ell n>n^{2}-n m-n+m-n^{2}+n+m n=m \geq 1 .
$$

So $\beta_{0}=0$. Hence, $g_{n-1}=y^{n-m-1} h_{m-1}$.

On the other hand, assume that $g_{n-1}$ is not divisible by $x$. Then setting $\hat{g}_{n-1}=\hat{g}_{n-1}(y)=g_{n-1}(0, y)$ we get that $\hat{g}_{n-1} \neq 0$ and satisfies (15) evaluated on $x=0$, that is $y \hat{g}_{n-1}=0$, which is not possible. Hence, $g_{n-1}$ is 
divisible by $x$. We write it as $g_{n-1}=x^{\ell} R_{\ell}$ with $1 \leq \ell<m-1$ and $R_{\ell}$ a polynomial of degree $n-1-\ell$ not divisible by $x$ and satisfying

$$
\begin{aligned}
& a x^{2} \frac{\partial R_{\ell}}{\partial x}+\frac{a m}{m-n} x y \frac{\partial R_{\ell}}{\partial y}-m x^{m-1-\ell} y^{n-m+1}+(n-m) x^{m-\ell} y^{n-m-1} \\
& =(y-a \ell x) R_{\ell} .
\end{aligned}
$$

Setting $\hat{R}_{\ell}=\hat{R}_{\ell}(y)=R_{\ell}(0, y)$, we have that $\hat{R}_{\ell} \neq 0$ and satisfies (16) restricted to $x=0$ that is $y \hat{R}_{\ell}=0$, which is not possible. So $g_{n-1}=x^{m-1} R_{\ell}$. We have also proved that $g_{n-1}=y^{n-m-1} h_{m-1}$. This means that

$$
g_{n-1}=x^{m-1} y^{n-m-1} Q_{1}=x^{m-1} y^{n-m-1}\left(\alpha_{1} x+\alpha_{2} y\right),
$$

with $\alpha_{1}, \alpha_{2}$ satisfying (see (15))

$$
\left(\alpha_{2}+m\right) y^{2}+\left(\alpha_{1}+a \alpha_{2}\right) x y+\left(\frac{a \alpha_{1} m+(m-n)^{2}}{m-n}\right) x^{2}=0 .
$$

Solving it we get $\alpha_{2}=-m, \alpha_{1}=a m$ and $a= \pm i(m-n) / m$ that is not possible because $a \in \mathbb{R}$. This completes the proof of Subcase 2.1.

Subcase 2.2.2: $c \neq 0$. System (4) becomes

$$
\begin{aligned}
& \dot{x}=-y+a x^{2}+b x y+c y^{2}, \\
& \dot{y}=x+\frac{a m}{m-n} x y+f y^{2} .
\end{aligned}
$$

Since there is at most two pairs of singular points at infinity we must have that

$$
\begin{aligned}
& y\left(a x^{2}+b x y+c y^{2}\right)-x\left(\frac{a m}{m-n} x y+f y^{2}\right) \\
& =\frac{y}{m-n}\left(-a n x^{2}+(b-f)(m-n) x y+c(m-n) y^{2}\right)=0
\end{aligned}
$$

has a unique double solution. If $a=0$ then $e=0$ and it follows from Theorem 10 (making the change $x \rightarrow y, y \rightarrow x, t \rightarrow-t$ ) that system (17) has at most one limit cycle. Then $a \neq 0$ and we get that

$$
c=\frac{(b-f)^{2}(n-m)}{4 a n},
$$

and the unique solutions of (18) are

$$
y=0 \quad \text { and } \quad y=\frac{2 a n}{(f-b)(n-m)} x,
$$

being the second solution a double solution. In this case

$$
g_{n}=y^{n-m}\left(y-\frac{2 a n}{(f-b)(n-m)} x\right)^{m} \text {. }
$$

Imposing that $g_{n}$ satisfies

$$
\left(a x^{2}+b x y+c y^{2}\right) \frac{\partial g_{n}}{\partial x}+\left(\frac{a m}{m-n} x y+f y^{2}\right) \frac{\partial g_{n}}{\partial y}-n y g_{n}=0,
$$

we get

$$
\frac{1}{2}(b m-f m-2 n+2 f n) y^{n-m+1}\left(y-\frac{2 a n}{(f-b)(n-m)} x\right)^{m}=0,
$$


which yields

$$
b=\frac{f m+2 n-2 f n}{m} .
$$

In short, in this case $c=(f-1)^{2} n(n-m) /\left(a m^{2}\right)$ and $b=(f m+2 n-2 f n) / m$.

If we pass to the coordinates $[X, Y, Z]$, the equilibrium points at infinity have all of them coordinate $X_{0} \neq 0$ so local coordinates $(u, v)$ with $u=Y / X$ and $v=Z / X$ are taken at these points in order to apply Theorem 12. Then system (4) with $c, e$ as before becomes

$$
\begin{aligned}
\dot{u}= & \frac{1}{a m^{2}(m-n)}\left(a^{2} m^{2} n u+a m^{2}(m-n) v+2 a(f-1) m(m-n) n u^{2}\right. \\
& \left.+(f-1)^{2}(m-n)^{2} n u^{3}+a m^{2}(m-n) v u^{2}\right) \\
\dot{v}= & -\frac{v}{a m^{2}}\left(a^{2} m^{2}+a(f m-2 f n+2 n) u m-(f-1)^{2}(m-n) n u^{2}\right. \\
& \left.-a u v m^{2}\right),
\end{aligned}
$$

and

$g(u, v)=u^{n-m}\left(u+\frac{a m}{(f-1)(m-n)}\right)^{m}+v g_{n-1}(u)+v^{2} g_{n-2}(u)+\cdots+v^{n} g_{0}(u)$,

where $g_{j}(u)$ is a polynomial of degree $j$, and the cofactor becomes

$$
k(u, v)=n u .
$$

The infinite equilibrium points for system (4) are on $v=0$ for system (19) with coordinates

$$
u=0 \text { (single point) or } u=\frac{a m}{(f-1)(n-m)} \text { (double point). }
$$

We take the double point $\left(u_{1}, 0\right)$ with $u_{1}=(a m) /((f-1)(n-m))$. Since $m \geq 1$ we have that $g\left(u_{1}, 0\right)=0$. Moreover the eigenvalues of the Jacobian matrix at that point are

$$
\mu=0, \quad \lambda=\frac{a m}{(f-1)(m-n)} .
$$

Since $\mu=0$, it follows from Theorem 12 (a) that either $s=2$ and $r=1$ in which case $k\left(u_{1}, 0\right)=\lambda$, or $s=1$ and $r=0$ in which case $k\left(u_{1}, 0\right)=\lambda$, or $s=$ $1, r=1$ in which case $k\left(u_{1}, 0\right)=0$. Since $k\left(u_{1}, 0\right)=($ anm $) /((f-1)(n-m))$ with $a m \neq 0$ we must have

$$
k\left(u_{1}, 0\right)=\frac{a n m}{(f-1)(n-m)}=\lambda=\frac{a m}{(f-1)(m-n)},
$$

which yields $n=-1$. So, this case is not possible.

Proof of Theorem 1 for system (4). In view of the proof of Theorem 14, the unique possible case to have an algebraic limit cycle lead to a system (4) having at most one limit cycle. This concludes the proof in this case. 


\section{Appendix: Quadratic Systems-Auxiliary RESUlt}

Lemma 15. Assume that $g(x, y)=0$ is an invariant algebraic curve $g=0$ of degree $n \geq 5$ with cofactor $k=$ ny of system

$$
\dot{x}^{\prime}=\beta_{1} x+\beta_{2} y+a x^{2}+b x y+c y^{2}, \quad \dot{y}=\beta_{3} x+\beta_{4} y+d x^{2}+e x y+f y^{2}
$$

for some $\beta_{1}, \beta_{2}, \beta_{3}, \beta_{4} \in \mathbb{R}$. If we write in sum of its homogeneous parts as $g=\sum_{k=0}^{n} g_{k}$ then

$$
\left(a x^{2}+(b-1) x y+c^{2}\right) \frac{\partial g_{n}}{\partial x}+\left(d x^{2}+e x y+(f-1) y^{2}\right) \frac{\partial g_{n}}{\partial y}=0,
$$

and for $k=n-1, \ldots, 0$,

$$
\begin{aligned}
& \left(\beta_{1} x+\beta_{2} y\right) \frac{\partial g_{k+1}}{\partial x}+\left(a x^{2}+(b-1) x y+c^{2}\right) \frac{\partial g_{k}}{\partial x}+\left(\beta_{3} x+\beta_{4} y\right) \frac{\partial g_{k+1}}{\partial y} \\
& +\left(d x^{2}+e x y+(f-1) y^{2}\right) \frac{\partial g_{k}}{\partial y}=(n-k) y g_{k} .
\end{aligned}
$$

Proof. Since $g(x, y)=0$ is an invariant algebraic curve $g=0$ of degree $n \geq 5$ with cofactor $k=n y$ of system (20) it must satisfy

$$
\left(\beta_{1} x+\beta_{2} y+\left(a x^{2}+b x y+c^{2}\right) \frac{\partial g}{\partial x}+\left(\beta_{3} x+\beta_{4} y+d x^{2}+e x y+f y^{2}\right) \frac{\partial g}{\partial y}=n y g .\right.
$$

Now we write $g=\sum_{j=0}^{n} g_{j}$ where each $g_{j}=g_{j}(x, y)$ is a homogeneous polynomial of degree $j$. Then computing the homogeneous parts in (23) we get

$$
\begin{aligned}
& \left(\beta_{1} x+\beta_{2} y\right) \frac{\partial g_{j+1}}{\partial x}+\left(a x^{2}+(b-1) x y+c^{2}\right) \frac{\partial g_{j}}{\partial x}+\left(\beta_{3} x+\beta_{4} y\right) \frac{\partial g_{j+1}}{\partial y} \\
& +\left(d x^{2}+e x y+(f-1) y^{2}\right) \frac{\partial g_{j}}{\partial y}=n y g_{j} .
\end{aligned}
$$

with the convention that $g_{n+1}=0$. Since $g_{j}$ must be a homogeneous polynomial of degree $j$ it must satisfy

$$
x \frac{\partial g_{j}}{\partial x}+y \frac{\partial g_{j}}{\partial y}=j g_{j}
$$

We rewrite (25) as

$$
x \frac{\partial g_{j}}{\partial x}+y \frac{\partial g_{j}}{\partial y}=n g_{j}+(j-n) g_{j}
$$

Now we multiply (26) by $y$ and we obtain

$$
x y \frac{\partial g_{j}}{\partial x}+y^{2} \frac{\partial g_{j}}{\partial y}=n y g_{j}+(j-n) y g_{j}
$$

So, introducing from (28) it yields

$$
n y g_{j}=x y \frac{\partial g_{j}}{\partial x}+y^{2} \frac{\partial g_{j}}{\partial y}+(n-j) y g_{j}
$$


Introducing (28) into (24), we get

$$
\begin{aligned}
& (\xi x-y) \frac{\partial g_{j+1}}{\partial x}+\left(a x^{2}+b x y\right) \frac{\partial g_{j}}{\partial x}+(x-\xi y) \frac{\partial g_{j+1}}{\partial y}+\left(d x^{2}+e x y+f y^{2}\right) \frac{\partial g_{j}}{\partial y} \\
& \left(\beta_{1} x+\beta_{2} y\right) \frac{\partial g_{j+1}}{\partial x}+\left(a x^{2}+(b-1) x y+c^{2}\right) \frac{\partial g_{j}}{\partial x}+\left(\beta_{3} x+\beta_{4} y\right) \frac{\partial g_{j+1}}{\partial y} \\
& \quad+\left(d x^{2}+e x y+(f-1) y^{2}\right) \frac{\partial g_{j}}{\partial y} \\
& =x y \frac{\partial g_{j}}{\partial x}+y^{2} \frac{\partial g_{j}}{\partial y}+(n-j) y g_{j},
\end{aligned}
$$

that is

$$
\begin{aligned}
& \left(\beta_{1} x+\beta_{2} y\right) \frac{\partial g_{j+1}}{\partial x}+\left(a x^{2}+(b-1) x y+c^{2}\right) \frac{\partial g_{j}}{\partial x}+\left(\beta_{3} x+\beta_{4} y\right) \frac{\partial g_{j+1}}{\partial y} \\
& +\left(d x^{2}+e x y+(f-1) y^{2}\right) \frac{\partial g_{j}}{\partial y}=(n-j) y g_{j} .
\end{aligned}
$$

as we wanted to prove.

\section{ACKNOWLEDGEMENTS}

We thank to the reviewer his/her comments which help us to improve the presentation of this paper.

The first author is partially supported by MINECO grants MTM201677278-P and MTM2013-40998-P, an AGAUR grant number 2014SGR-568, and the grant FP7-PEOPLE-2012-IRSES 318999. The second author is partially supported by FCT/Portugal through the project UID/MAT/04459/2013.

\section{REFERENCES}

[1] M. Abramowitz And I.A. Stegun, Handbook of mathematical functions with formulas, graphs and mathematical tables, National Bureau of Standards Appl. Math. Series $\mathbf{5 5}, 1964$.

[2] E. Brieskorn, H. KnÖrer, Plane Algebraic Curves, Birkhäuser-Verlag, Basel, 1986.

[3] J. Chavarriga, H. Giacomini And M. Grau, Necessary conditions for the existence of invariant algebraic curves for planar polynomial systems, Bull. Sci. Math. 129 (2005), 99-126.

[4] J. Chavarriga, H. Giacomini and J. Llibre, Uniqueness of algebraic limit cycles for quadratic systems, J. Math. Anal. Appl. 261 (2001), 85-99.

[5] J. ChAVARRIGA AND J. LliBRE, Invariant algebraic curves and rational first integrals planar polynomial vector fields, J. Differential Equations 169 (2001), 1-16.

[6] J. Chavarriga, J. Llibre and J. Sorolla, Algebraic limit cycles of degree four for quadratic systems, J. Differential Equations 2009 (2004), 206-244.

[7] L.S. Chen, Uniqueness of the limit cycle of a quadratic system in the plane, Acta Mathematica Sinica, (in chinese) 20 (1977), 11-13.

[8] C. Christopher, Invariant algebraic curves and conditions for a center, Proc. Roy. Soc. Edinburhgh. 124A (1994), 1209-1229.

[9] C. Christopher, J. Llibre And G. Swirszcz, Invariant algebraic curves of large degree for quadratic systems, J. Math. Anal. Appl. 303 (2005), 206-244.

[10] B. Coll AND J. LliBRe, Limit cycles for a quadratic systems with an invariant straight line and some evolution of phase portraits in Qualitative Theory of Differential Equations, Colloquia Mathematica Societatis Janos Bolyai, 53, Bolyai Institute, Hungary, 1988, 111-123. 
[11] B. Coll, G. Gasull And J. Llibre, Quadratic systems with a unique finite rest point Publicacions Matematiques 32 (1988), 199-259

[12] W.A. Coppel, Some quadratic systems with at most one limit cycle Dynamics Reported, Vol. 2, 1989, 61-88.

[13] R.M. Evdokimenco, Construction of algebraic paths and the qualitative investigation in the large of the properties of integral curves of a system of differential equations, Differential Equations 6 (1970), 1349-1358.

[14] R.M. Evdokimenco, Behavior of integral curves of a dynamic system, Differential Equations 9 (1974), 1095-1103.

[15] R.M. Evdokimenco, Investigation in the large of a dynamic systems with a given integral curve, Differential Equations 15 (1979), 215-221.

[16] V.F. Filiptsov, Algebraic limit cycles, Differential Equations 9 (1973), 983-986.

[17] D. Hilbert, Mathematische Probleme, in: Lecture, Second Internat. Congr. Math., Paris, 1900, in: Nachr. Ges. Wiss. Göttingen Math.-Phys. Kl., 1900, pp. 253?297; English transl. in: Bull. Amer. Math. Soc. 8 (1902) 4370-479.

[18] J. Llibre, Integrability of polynomial differential systems, Handbook of Differential Equations, Ordinary Differential Equations, Eds. A. Cañada, P. Drabek and A. Fonda, Elsevier, Vol. 1, 2004, pp 437-533.

[19] J. LliBRE, Open problems on the algebraic limit cycles of planar polynomial vector fields, Bull. of Academy of Sciences of Moldova (Matematica), vol. 56 (2008), 19-26.

[20] J. Llibre And C. PANtazi, Counterexample to a conjecture on the algebraic limit cycles of polynomial vector fields, Geometriae Dedicata 110 (2005), 213-219.

[21] J. Llibre, R. Ramírez And N. Sadovskaia, On the 16th Hilbert problem for algebraic limit cycles, J. Differential Equations 248 (2010), 1401-1409.

[22] J. Llibre, R. RamíRez And N. Sadovskaia, On the 16th Hilbert problem for limit cycles on nonsingular algebraic curves, J. Differential Equations 250 (2011), 983-999.

[23] J. Llibre AND G. Swirszcz, Classification of quadratic systems admitting the existence of an algebraic limit cycle, Bulletin des Sciences Mathemàtiques 131 (2007), 405-421.

[24] J. LliBRE AND C. VALLS, Quadratic polynomial differential systems with one pair of singular points at infinity have at most one algebraic limit cycle, preprint, see the web page http://www.gsd.uab.cat/.

[25] B. SHEn, The problem of the existence of limit cycles and separatrix cycles of cubic curves in quadratic systems, (Chinese) Chinese Ann. Math. Ser. A 12 (1991), no. 3, 382-389.

[26] A.I. YABLONSKiI, Limit cycles of a certain differential equations, Differential Equations 2 (1966), 193-239.

[27] Q. YuAn-Xun, On the algebraic limit cycles of sencod degree of the differential equation $d y / d x=\sum_{0 \leq i+j \leq 2} a_{i j} x^{i} y^{j} / \sum_{0 \leq i+j \leq 2} b_{i j} x^{i} y^{j}$, Acta Math. Sinica 8 (1958), 23-35.

[28] X. Zhang, Invariant algebraic curves and rational first integrals of holomorphic foliations in CP(2), Sci. China Ser. A 46 (2003), no. 2, 271-279.

[29] X. ZhANG, The 16th Hilbert problem on algebraic limit cycles, J. Differential Equations 251 (2011), 1778-1789.

${ }^{1}$ Departament de Matemàtiques, Universitat Autònoma de Barcelona, 08193 Bellaterra, Barcelona, Catalonia, Spain

E-mail address: jllibre@mat.uab.cat

Departamento de Matemática, Instituto Superior Técnico, Universidade de Lisboa, 1049-001 Lisboa, Portugal

E-mail address: cvalls@math.ist.utl.pt 